\title{
Corela
}

Cognition, représentation, langage

HS-19 | 2016

Le point de vue pris au mot

\section{Évaluation et gradation dans une décision de la cour européenne des droits de l'homme}

\section{Montserrat Cunillera Domènech}

\section{(2) OpenEdition}

Journals

\section{Édition électronique}

URL : http://journals.openedition.org/corela/4357

DOI : 10.4000/corela.4357

ISSN : 1638-573X

\section{Éditeur}

Cercle linguistique du Centre et de l'Ouest - CerLICO

\section{Référence électronique}

Montserrat Cunillera Domènech, «

Évaluation et gradation dans une décision de la cour européenne des droits de l'homme », Corela [En ligne], HS-19 | 2016, mis en ligne le 08 juin 2016, consulté le 01 mai 2019. URL : http://

journals.openedition.org/corela/4357 ; DOI : 10.4000/corela.4357

Ce document a été généré automatiquement le 1 mai 2019.

\section{(i) (2)}

Corela - cognition, représentation, langage est mis à disposition selon les termes de la licence Creative Commons Attribution - Pas d'Utilisation Commerciale - Partage dans les Mêmes Conditions 4.0 International. 


\section{Évaluation et gradation dans une décision de la cour européenne des droits de l'homme}

Montserrat Cunillera Domènech

\section{Introduction}

1 En tant qu'instruments de régulation de la vie sociale et économique, les textes juridiques sont étroitement liés à une communauté linguistique et culturelle déterminée et reflètent le rapport de forces qui s'établit entre ses membres. Ce sont des textes ${ }^{2}$ qui se veulent objectifs et neutres (Duarte et Martínez 1995) mais qui n'échappent pas pour autant à la présence d'unités signalant l'intervention du locuteur (Gotti 2008; Cunillera 2012 ; Serpa 2013). Ainsi, la présente étude s'inscrit dans la lignée des travaux de recherche qui entendent montrer que les textes juridiques contiennent, malgré toute volonté d'objectivité, des marques qui laissent entendre la voix du locuteur et son point de vue à l'égard de ce qu'il rapporte.

2 L'analyse d'un arrêt de la Cour européenne des droits de l'homme (désormais CEDH) devrait permettre d'illustrer cette tension entre objectivité et subjectivité propre à la plupart des textes juridiques, car c'est un texte représentatif d'un genre juridique : la jurisprudence (Borja Albi 2007). L'intérêt pour ces textes repose sur leur nature discursive et énonciative: malgré leur caractère argumentatif, ils cherchent à dissimuler toute marque de subjectivité et à faire passer la décision prise par la Cour comme impartiale et objective. Cependant, dans le déploiement de son argumentation, la Cour laisse entrevoir - d'une manière subtile mais inévitable - sa propre évaluation des faits. Nous interrogerons la mise en place de cette évaluation: Quels sont les mécanismes linguistiques qui révèlent, dans ce type de textes, la présence du locuteur et son 
positionnement par rapport à des voix alternatives? Pour mener notre analyse, nous ferons appel à la théorie de l'Appraisal (Martin et White 2005) et à la Sémantique des Points de Vue (Raccah 2005a, 2005b), les deux étant utiles pour notre objet d'étude, car elles s'occupent des effets que les choix linguistiques du locuteur provoquent dans la construction du sens textuel. Le but final de cette approche sur l'évaluation et son expression, c'est de déceler quelques pistes sur le fonctionnement discursif et argumentatif des textes qui représentent la voix de la justice.

\section{Corpus}

Comme nous le savons, un arrêt est une décision de justice d'une Cour et, du point de vue des genres juridiques, c'est un texte qui relève de la jurisprudence (Borja Albi 2007), donc qui crée du droit dans la culture juridique où il s'inscrit. En général, ces textes sont très complexes en raison de leur nature hybride, car un arrêt est construit à partir d'autres textes juridiques (il contient des fragments des lois, du pourvoi, d'autres arrêts, etc.), et de son caractère polyphonique, étant donné qu'il incorpore le discours de plusieurs locuteurs et énonciateurs (Ducrot 1984). En même temps, ce sont des textes très variés dans le sens qu'un arrêt peut présenter une diversité de situations juridiques (selon le type de pourvoi, le type de décision prise par la Cour - cassation ou rejet, etc.) et une grande variété thématique (les branches du droit où un arrêt peut s'inscrire sont nombreuses ainsi que le sont les différentes situations de la vie quotidienne auquel il renvoie).

4 L'arrêt sur lequel portera notre analyse fut prononcé le 7 octobre 2014 par la Troisième Section de la Cour européenne des droits de l'homme ${ }^{3}$; par conséquent, il fait partie de la jurisprudence de la CEDH sur les droits fondamentaux des personnes, celle-ci ayant une grande influence sur le droit européen ${ }^{4}$. Intitulé "Affaire Etxebarria Caballero c. Espagne ", l'arrêt est publié dans le web de cette Cour ${ }^{5}$ et son contenu peut être résumé de la façon suivante : la requérante saisit la Cour européenne pour dénoncer la violation de l'article 3 de la Convention européenne des droits de l'homme, qui interdit aux États de pratiquer la torture ou de soumettre une personne relevant de sa juridiction à des peines ou des traitements inhumains ou dégradants. Pour démontrer la violation de cet article par les autorités espagnoles, la requérante présente deux allégations: l'insuffisance des investigations menées par les autorités espagnoles et les mauvais traitements prétendument subis lors de sa détention. La première allégation invoque la violation de l'article 3 de la Convention dans son volet procédural et la deuxième, dans son volet matériel. La Cour a accueilli la première allégation, la résolution a donc été favorable à la requérante, alors que la deuxième allégation a été rejetée, en conséquence, la résolution a été défavorable à la requérante et favorable aux autorités espagnoles.

Pour nous approcher des éléments linguistiques qui expriment l'évaluation et l'intervention de la Cour européenne dans le texte, nous examinerons surtout la réponse de la Cour aux allégations de la requérante. Sa réponse est exposée au long des deux sections intitulées "L'appréciation de la Cour ». La Figure $\mathrm{n}^{\circ} 1$ ci-dessous montre un schéma de la partie de l'arrêt qui nous intéresse et permet d'identifier chacune de ces sections prévisiblement plus évaluatives : 


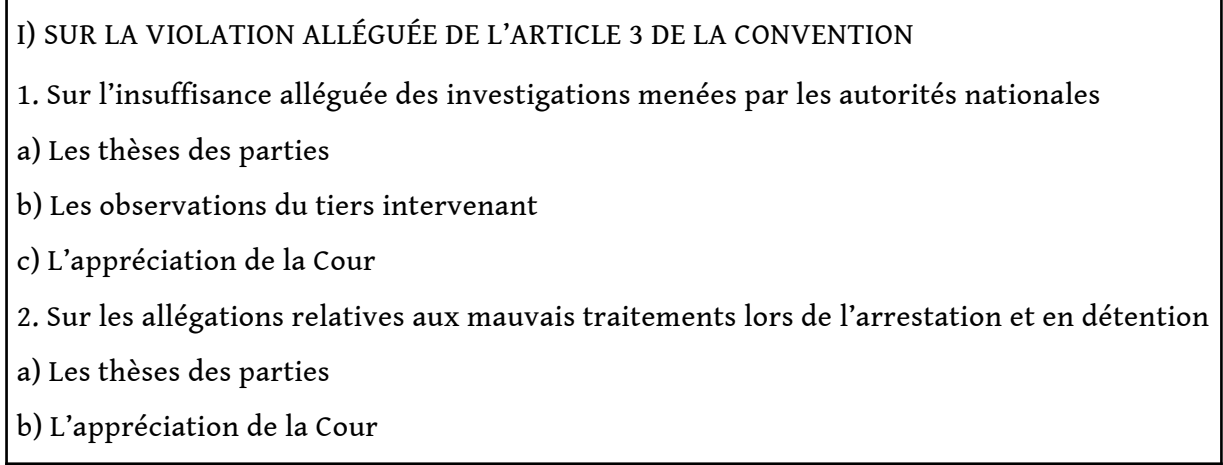

Figure $n^{0} 1$ : Structure de la première branche de l'arrêt

6 Dans les sections marquées la Cour reprend les arguments des parties - la requérante et les autorités espagnoles -, et en même temps elle développe ses propres opinions sur ces arguments. Naturellement, ces opinions, ainsi que toute décision judiciaire, ne peuvent se fonder que sur la loi et la jurisprudence, elles doivent être donc motivées et se présenter comme objectives et impartiales. Ce sont ces sections qui vont nous fournir la plupart de données en rapport avec la façon dont sont exprimées les évaluations de la Cour. Avant d'exposer notre analyse, il convient de préciser le cadre théorique où elle s'inscrit.

\section{Outillage conceptuel}

7 La théorie descriptive de la Sémantique des Points de Vue (SPV) de Raccah (2005a, 2005b), héritière de la Théorie de l'Argumentation dans la langue (TAL) d'Anscombre et Ducrot (1983), s'est révélée pertinente pour l'analyse du discours parce qu'elle permet de rendre compte des instructions essentielles des unités lexicales et de leurs effets dans la construction du sens. Toute unité linguistique est porteuse d'un faisceau d'instructions sémantico-pragmatiques qui imposent une certaine orientation argumentative et reflètent un point de vue spécifique de la part du locuteur. Ainsi, les mots et les structures d'une langue reflètent des appréciations, favorables ou défavorables, à propos d'une chose, d'une personne ou d'une attitude, de la part du locuteur lors de l'énonciation et à propos de la situation concernée par l'énonciation (Anscombre et Ducrot 1983). Selon Ducrot (1984 : 205), le locuteur donne existence « à des énonciateurs dont il organise les points de vue et les attitudes » et la position du locuteur peut se manifester à travers son engagement ou désengagement vis-à-vis des points de vue attribués aux différents énonciateurs. Les notions d'orientation argumentative, point de vue et instruction sémantique de la TAL ont été reprises et développées par la SPV pour définir ses propres objectifs :

Se fondant sur le fait qu'une argumentation vise à faire adopter un point de vue tout en supposant admis d'autres points de vue, la Sémantique des Points de Vue propose de décrire dans un système unifié et les orientations argumentatives et les points de vue, assignant pour tâche à la sémantique de décrire les contraintes que les unités linguistiques imposent aux points de vue visés par les énoncés, mais aussi aux points de vue supposés par ces énoncés. (Raccah 2008 : 79)

8 Raccah (2005a) établit une distinction entre les mots euphoriques et les mots dysphoriques pour désigner les jugements associés aux mots dans n'importe quelle situation. Selon cet auteur, les unités euphoriques sont celles qui véhiculent des points de vue toujours 
positifs, et les dysphoriques, celles qui expriment des points de vue toujours négatifs. Les mots qui ne sont ni euphoriques ni dysphoriques n'ont pas d'instruction explicite, mais cela ne signifie pas qu'ils ne puissent pas montrer une évaluation positive ou négative selon le contexte et l'idéologie de l'énonciateur : «嚂...區 le jugement évoqué par ces mots peut être positif ou négatif, mais il est contraint par le jugement que l'interprète porte sur d'autres entités que celle que le mot évoque » (Raccah 2005b : 176).

Ce sont aussi les notions de point de vue, attitude et voix qui sont au cœur de la théorie de l' Appraisal ou Théorie de l'Évaluation (Martin et White 2005; White 2003). Cette théorie, issue de la linguistique systémique fonctionnelle, s'intéresse aux marques linguistiques qui laissent entrevoir la présence subjective du sujet parlant dans le texte, son positionnement par rapport aux destinataires et son système d'évaluation (Martin et White $2005: 164)$. Elle propose un système de classification des effets créés par les différents choix du locuteur, classification qui est organisée en trois sous-systèmes interdépendants :

- l'AtTitude, qui permet de décrire les réactions émotionnelles et les opinions ;

- l'engagement, qui concerne le degré d'acceptation par le locuteur de sa proposition et la manière dont celui-ci négocie avec les points de vue présentés dans d'autres énoncés (réels ou supposés);

- la GRADATION, qui rend compte de l'intensité des évaluations exprimées, ainsi que du degré avec lequel les propositions sont modulées par l'engagement.

Dans la présente étude, pour analyser l'expression de l'évaluation dans un arrêt de la CEDH, nous appliquerons la théorie de l'Appraisal, en nous centrant sur les systèmes de l' ATTITUDE et de la GRADATION ${ }^{6}$. Les stratégies de ces deux systèmes ont sans doute une incidence sur la force argumentative du discours telle que la conçoit la Sémantique des Points de Vue, le deuxième pilier théorique qui soutient notre analyse.

\section{L'expression de l'ATTITUDE et la GRADATION dans un arrêt de la CEDH}

11 La Cour doit statuer sur les deux allégations qui sont à l'origine de la procédure judiciaire et qui portent sur le comportement des autorités espagnoles à l'égard de la requérante et sur la situation vécue par la requérante lors de son arrestation et sa détention. Tout au long de son argumentation, la voix de l'autorité judiciaire reprend chaque allégation et, avant de parvenir à la résolution finale, elle laisse entendre son évaluation à propos des contenus idéationnels. Le comportement des autorités est évalué indirectement à travers l'évaluation de l'enquête et les mesures de surveillance juridictionnelle exigées par la loi. La situation vécue par la requérante est évaluée, à son tour, à travers l'appréciation des preuves et de la gravité des faits. Nous allons étudier la façon particulière dont sont exprimées les évaluations de ces contenus.

\subsection{L'ATTITUDE : I'Appréciation}

Le système de l'ATTITUDE est divisé en trois sous-systèmes :

- l'Affect qui concerne les émotions ;

- le Jugement qui s'occupe de l'évaluation du comportement humain en rapport avec les normes sociales ; 
- l'Appréciation qui évalue d'autres entités comme des choses, des actuations ou des phénomènes.

14 L'expression de l'évaluation peut être directe, explicite, à travers des unités lexicales et des constructions grammaticales évaluatives, ou bien indirecte, quand les choix linguistiques laissent entrevoir des évaluations liées aux valeurs, aux connaissances et aux points de vue partagés par une communauté culturelle.

15 En tenant compte de la nature des éléments qui sont évalués dans notre corpus (l'enquête, les mesures de surveillance, etc.), c'est la catégorie de l'Appréciation qui est concernée. Pour exprimer ses appréciations, la Cour utilise des unités lexicales sémantiquement très vagues et imprécises, et apparemment neutres dans le sens que seulement le contexte permet d'identifier l'actualisation d'une valeur positive ou négative; elles reflètent donc une volonté d'objectivité dans la présentation des faits rapportés et de l'application du droit. La Cour emploie ce type d'unités pour parler de l'enquête, les investigations et les mesures de surveillance juridictionnelle mises en place par les autorités espagnoles vis-à-vis de celles qui sont exigées par la loi. Les unités lexicales employées pour répondre à la deuxième allégation de la requérante sont aussi sémantiquement vagues et en apparence objectives: gravité, preuves. Ces unités sont utilisées pour faire référence aux deux arguments susceptibles, d'après la Cour, d'établir l'existence des mauvais traitements contre quelqu'un. Enfin, des unités lexicales plus précises ont été réservées pour détailler les traitements dénoncés par la requérante.

\subsubsection{Imprécision sémantique et apparente objectivité}

- Les adjectifs effectif/ve et approfondi / e

16 Ils ont été choisis pour parler de l'enquête exigée par la loi et de l'enquête et les investigations mises en place par les autorités espagnoles. Ces adjectifs apparaissent dans un nombre élevé d'occurrences ( 6 occurrences d'effectif et 4 d'approfondi). Par exemple :

(1) À la lumière des éléments qui précèdent, la Cour estime que l'enquête menée dans la présente affaire n'a pas été suffisamment approfondie et effective pour remplir les exigences précitées de l'article 3 de la Convention'.

Conscient peut-être de cette imprécision, le locuteur introduit une définition mais elle est aussi très vague d'un point de vue sémantique, car elle contient des termes également indéterminés comme justifié, raisonnable, etc. ${ }^{8}$. Les adjectifs sont la principale forme que peut prendre l'Attitude, et aussi la façon la plus naturelle d'exprimer une évaluation (Gardin 2009: 2), mais dans l'arrêt, il semble qu'ils ne véhiculent pas d'évaluations explicites.

18 Les adjectifs effective et approfondie peuvent avoir un sens positif ou négatif selon le contexte; dans l'arrêt analysé et par rapport à l'enquête qui a dû être examinée, ils ont une valeur positive, car ils orientent l'énoncé vers une conclusion du type [ÊTRE CONFORME AU DROIT / À LA LOI]. Cette conclusion est positive dans le domaine de la justice, ainsi que dans la société et la culture où le texte s'inscrit. Le discours de la Cour présente une vision qui souligne l'importance de la légalité.

- Les adjectifs précis, circonstancié, détaillé

19 Ils ont été employés pour définir des actions et des procès. Ils sont utilisés notamment quand la Cour se prononce sur le comportement de la requérante - sur la manière dont elle a présenté sa plainte - et sur l'exposition des faits (des mauvais traitements) de la deuxième allégation, qui n'a pas eu une résolution favorable. Ces unités lexicales 
exprimant une idée de précision et de détail actualisent un sens positif dans ce contexte, parce qu'elles orientent les énoncés vers une conclusion du type [ÊTRE CONFORME À LA LOI], d'une façon semblable aux adjectifs précédents. Par exemple :

(2) L'intéressée s'est plainte de manière précise et circonstanciée d'avoir fait l'objet de mauvais traitements au cours de sa garde à vue au secret : le 5 mars 2011 lorsqu'elle a été traduite devant le juge central d'instruction de l'Audiencia Nacional et une seconde fois le 15 mars 2011 lorsqu'elle a porté plainte devant la juge d'instruction $\mathrm{n}^{\circ} 1$ de Bilbao.

(3) La Cour note que, dans sa requête, la requérante a exposé de manière détaillée et circonstanciée les sévices dont elle dit avoir été victime durant son arrestation et détention.

- Des substantifs tels que isolement, communication avec l'extérieur ou vulnérabilité

Ils ont été utilisés dans la description que la Cour fait de la situation vécue par la requérante lors de son arrestation et détention. Ce ne sont pas des unités explicitement évaluatives, mais elles ont un potentiel évocateur élevé dans la culture où elles s'inscrivent (elles sont associées à des mauvais traitements, à une idée de douleur, d'atteinte contre la personne, etc.) et elles actualisent une valeur négative dans le contexte analysé. Parfois la négativité provient des éléments du cotexte qui expriment une gradation comme absence totale dans le segment absence totale de communication (que nous reprendrons par la suite, cf. § 4.2). L'ensemble de ces unités lexicales a un effet indirect sur l'évaluation du comportement des autorités espagnoles : implicitement elles mènent à une conclusion du type [LES AUTORITÉS ESPAGNOLES N'ONT PAS AGI CONFORMÉMENT À LA LOI], donc les autorités sont évaluées négativement.

- Des lexèmes comme approprié et appliqué

21 Ils ont été choisis pour parler des mesures de surveillance juridictionnelle. Leur apparente neutralité sémantique et argumentative est de nouveau nuancée par la présence d'éléments de gradation (rigoureusement, etc.), qui montrent l'intervention du locuteur :

(4) Elle estime que la situation de vulnérabilité particulière des personnes détenues au secret commande que soient imposées par le code de procédure pénale des mesures de surveillance juridictionnelle appropriées et que celles-ci soient rigoureusement appliquées, [...]

\subsubsection{Les unités lexicales sémantiquement précises}

Elles ont été utilisées pour mentionner les traitements dénoncés par la requérante dans la deuxième allégation. La plupart de ces unités sont dysphoriques, car elles sont négatives dans n'importe quelle situation :

- mauvais traitements, séances d'asphyxie, humiliations et vexations sexuelles, menaces, etc.

D'autres lexèmes, ont une valeur négative dans ce contexte.

- coups sur la tête, cris dans les oreilles, frappes et secousses, etc.

La description des faits à l'aide de ces termes offre une vision négative de la situation subie par la requérante et, indirectement, une évaluation négative du comportement des autorités responsables. Cependant, l'adhésion de la Cour à cette évaluation négative est immédiatement invalidée par l'emploi du conditionnel passé, auraient revêtu, qui marque une distanciation énonciative. La désinence verbale apporte une idée de virtualité et exprime un degré d'intensité faible du point de vue de l'ENGAGEMENT'. Dans l'arrêt, la Cour 
utilise donc le mode conditionnel pour attribuer des énoncés à la voix de la requérante et montrer qu'elle ne les assume pas ${ }^{10}$.

\subsection{La gradation}

Au moyen de la GRADATION le locuteur peut préciser le sens et la portée des évaluations et des affirmations, en modifiant l'intensité ou la spécificité de ce qui a été dit (Hood et Martin 2005 : 200 ; Navarro $2014: 17$ ). Ce système comprend deux sous-systèmes :

- la FORCE, qui correspond à une gradation en fonction de l'intensité (de l'opinion émise) ou de la quantité ;

- le focus, qui modélise le degré de prototypicalité des termes employés (Gardin 2009).

Dans la présente étude, nous n'analyserons que le premier sous-système. La FORCE peut s'exprimer de deux manières :

- à partir de la QuAnTification du numéro (beaucoup), de la masse (petit) et de l'extension (bref);

- à travers l'inTENSIFICATION des qualités (très mauvais), des procès (observer rapidement) ou des propositions (doit considérer) (Navarro $2014: 17$ ).

Dans notre corpus, l'expression de l'évaluation de la Cour au moyen d'un grand nombre d'unités lexicales sémantiquement imprécises (§ 3.1.) est compensée par la présence d'autres options linguistiques qui laissent entrevoir la subjectivité du locuteur et qui relèvent du système de la GRADATION. Ainsi, on a constaté plusieurs éléments lexicaux et grammaticaux liés à la catégorie de la FORCE qui graduent les Appréciations mises en place par le locuteur, soit à travers l'expression de la QUANTITÉ (pour les entités concrètes et abstraites) soit à travers l'INTENSITÉ (pour les qualités et les procès).

Les lexèmes qui apportent de la FORCE aux énoncés en exprimant la QUANTITÉ des choses sont:

- suffisamment, insuffisance, un minimum et absence

Ces unités accompagnent les lexèmes imprécis mentionnés ci-dessus aussi bien pour parler de l'enquête et les investigations des autorités (effective, approfondie, etc.) que pour décrire la situation vécue par la requérante lors de son arrestation et détention (gravité, preuves).

L'adverbe modificateur suffisamment et le nom insuffisance introduisent l'idée d'un degré minimum nécessaire pour que l'enquête puisse être considérée régulière et légale. La négation ne pas pour nier suffisamment et le préfixe négatif in- (dans insuffisance) indiquent que ce degré minimum n'a pas été atteint et, par conséquent, la conclusion à laquelle mènent les énoncés assumés par la Cour à propos de l'enquête est négative (donc indirectement l'évaluation des autorités, elle aussi, est négative) :

(5) [...] l'enquête n'as pas été suffisamment approfondie et effective [...]

(6) Cependant, en raison de l'absence d'éléments probatoires suffisants résultant notamment de l'insuffisance de l'enquête menée, la Cour ne s'estime pas en mesure d'affirmer [...]

31 Dans l'énoncé Sur l'insuffisance alléguée des investigations menées par les autorités nationales, la forme nominale insuffisance apportait une évaluation explicite négative avec un degré d'intensité élevé mais simultanément la Cour s'en éloignait à l'aide du participe alléguée et l'attribuait à un autre locuteur (la requérante). En revanche, dans l'exemple (6) la Cour 
reprend le segment évaluatif l'insuffisance de l'enquête et montre son acceptation car le lexème alléguée n'est plus présent.

Ces unités de QUANTITÉ introduisent aussi l'idée d'un degré minimum nécessaire pour que les preuves et la gravité des faits (deuxième allégation) puissent être considérés valables pour aboutir à une conclusion concrète :

(7) [...] éléments de preuve appropriés ou faisceau d'indices ou des présomptions non réfutées, suffisamment graves, précis et concordants [...]

(8) [...] la Cour considère que les éléments dont elle dispose ne lui permettent pas d'établir, au-delà de tout doute raisonnable, que la requérante a été soumise à des traitements ayant atteint un minimum de gravité, en méconnaissance de l'article 3 de la Convention [...]

L'unité nominale absence intensifie l'idée d'insuffisance apportée par les unités précédentes parce qu'elle signifie la négation absolue :

(9) En conclusion, eu égard à l'absence d'enquête approfondie et effective au sujet des allégations défendables de la requérante (Martinez Sala et autres $c$. Espagne, no $58438 / 00, \& 156-160,2$ novembre 2004), selon lesquelles elle avait subi des mauvais traitements au cours de sa garde à vue, la Cour estime qu'il y a eu violation de l'article 3 de la Convention dans son volet procédural.

Dans l'exemple (9) la négation absolue apportée par le mot absence concerne non seulement les adjectifs approfondie et effective qui accompagnent enquête mais aussi le substantif lui-même. L'unité absence renforce la valeur négative qui dérive de l'appréciation initiale (l'enquête n'as pas été suffisamment approfondie et effective), et ainsi, désormais, l'enquête est présentée non seulement comme insuffisante mais en plus comme inexistante. Le même effet de renforcement se produit dans les exemples cidessous, car l'unité absence nie et annule complètement la possibilité d'une situation favorable lorsque la requérante était retenue par les autorités espagnoles en (10) ainsi que l'existence d'éléments probatoires en (11). En (10), l'adjectif totale intensifie encore plus l'évaluation négative évoquée par le lexème absence à propos de la situation vécue par la requérante :

(10) [...] la requérante se trouvait, pendant la période de temps où les mauvais traitements allégués se seraient produits, dans une situation d'isolement et $d$ ' absence totale de communication avec l'extérieur [...]

(11) Cependant, en raison de l'absence d'éléments probatoires suffisants résultant notamment de l'insuffisance de l'enquête menée, la Cour ne s'estime pas en mesure d'affirmer avec le degré de certitude voulu par sa propre jurisprudence que la requérante a été soumise, lors de son arrestation et de sa détention, aux mauvais traitements allégués.

En ce qui concerne la deuxième catégorie de la FORCE, l'INTENSITÉ, on a constaté plusieurs mécanismes. Le premier comprend des éléments qui relèvent de la QUALITÉ (degré) comme les éléments comparatifs :

-un effort plus important (ce que les autorités auraient dû faire et n'ont pas fait), d'autant plus fortement, etc.

36 Quand une description apparemment objective est intensifiée, on doit l'interpréter de façon évaluative (Hood et Martin 2005: 208). Le second mécanisme d'intensité est constitué par l'accumulation de lexèmes ayant un sens similaire (Martin et White 2005 : 144 ; Serpa $2013: 13)$ :

(12) [...] une telle preuve peut néanmoins résulter d'un faisceau d'indices, ou de présomptions non réfutées, suffisamment graves, précis et concordants [...] 

utilisés surtout pour parler de l'enquête et les mesures de surveillance juridictionnelle exigées par la loi en opposition à celles mises en place par les autorités espagnoles.

- commander, s'imposer, exiger

Ces verbes apportent de l'intensité parce qu'ils montrent une nécessité, un devoir et se situent donc du côté de la PROPOSITION. Ils expriment une obligation forte qui, comme on découvre par le contexte, ne s'est pas accomplie :

(13) [...] la situation de vulnérabilité particulière des personnes détenues au secret commande que soient imposées par le code de procédure pénale des mesures de surveillance juridictionnelle appropriées et que celles-ci soient rigoureusement appliquées, [...]

(14) Une investigation effective s'impose pourtant d'autant plus fortement lorsque, comme en l'espèce, la requérante se trouvait, pendant la période de temps où les mauvais traitements allégués se seraient produits, dans une situation d'isolement et d'absence totale de communication avec l'extérieur, pareil contexte exigeant un effort plus important, de la part des autorités internes, pour établir les faits dénoncés [...]

Le quatrième mécanisme d'intensité est formé par des adverbes comme

- rigoureusement, particulièrement, notamment ou fortement

qui sont en rapport avec le PRocÈs et des adjectifs comme

- particulière

41 (dans vulnérabilité particulière) qui sont en rapport avec la QUALITÉ pour parler de la situation de la requérante détenue. L'adverbe rigoureusement apporte de l'intensité à d'autres lexèmes sémantiquement imprécis (appropriées, appliquées) utilisés pour évaluer les mesures de surveillance juridictionnelle des autorités espagnoles. L'adverbe fortement vient renforcer le verbe s'imposer qui, comme nous avons déjà vu, est porteur lui-même d'un degré élevé d'intensité. Ces deux adverbes renforcent l'idée de nécessité ou le devoir de réaliser une action déterminée :

(15) Une investigation effective s'impose pourtant d'autant plus fortement lorsque, comme en l'espèce, la requérante se trouvait, pendant la période de temps où les mauvais traitements allégués se seraient produits [...]

Enfin, le verbe restrictif se borner à apporte une atténuation de l'intensité (PROCÈs), qui dans ce contexte est perçue comme étant négative parce qu'elle oriente l'énoncé vers une conclusion du type [CE N'EST PAS SUFFISANT POUR ÊTRE CONSIDÉRÉ CONFORME À LA LOI]. Il laisse sous-entendre que les autorités n'ont pas fait tout ce qu'elles auraient dû faire ou qu'elles auraient pu faire. Cet argument s'oppose à l'énoncé suivant qui explicite toutes les demandes de la requérante, l'opposition étant soulignée par le connecteur argumentatif alors que :

(16) [...] la juge d'instruction no 1 de Bilbao s'est bornée à examiner les rapports des médecins légistes et les copies des dépositions de la requérante alors que cette dernière avait aussi sollicité la production des enregistrements des caméras de sécurité des locaux [...]

Dans l'arrêt analysé, les appréciations négatives n'ont pas été déployées par l'emploi de lexèmes explicitement évaluatifs mais par l'emploi de lexèmes exprimant la GRADATION, donc indirectement. Dans la première allégation, l'insuffisance de l'enquête et des mesures de surveillance juridictionnelle et l'obligation de les mettre en place impliquent une évaluation négative du comportement des autorités espagnoles. L'argumentation de la Cour est fondée sur ces deux éléments juridiques qui mènent à conclure qu'il y a eu 
vulnération de l'article 3 de la Convention dans son volet procédural (résolution favorable à la requérante). Et dans la deuxième allégation, on retrouve les mêmes arguments en termes d'insuffisance pour parler des preuves et de la gravité des faits. Ils construisent une évaluation négative indirecte et permettent de conclure qu'il n'y a pas eu vulnération de l'article 3 de la Convention dans son volet matériel (résolution défavorable à la requérante).

\section{Conclusion}

L'ensemble des mécanismes linguistiques analysés dans notre corpus participent à la construction des effets d'objectivité et montrent en même temps un positionnement subjectif déterminé. Ainsi, l'autorité judiciaire expose sa décision en évitant l'emploi d'un lexique évaluatif explicite mais, en même temps, des lexèmes apparemment neutres sont précisés à l'aide des stratégies relevant de la GRADATION, surtout de la FORCE. Les stratégies de GRADATION - FORCE évaluent les contenus idéationnels d'une manière indirecte; le locuteur les situe subjectivement dans une escale qui implique des valeurs relatives. Plusieurs éléments relevant du sous-système de la FORCE et qui expriment la QUANTITÉ et l' INTENSITÉ contribuent à renforcer une évaluation négative ou à la construire. En l'espèce, l'évaluation négative porte aussi bien sur l'enquête et les mesures de surveillance juridictionnelle - de la première allégation - que sur les preuves de l'existence des traitements dénoncés par la requérante - dans la deuxième allégation. En termes de la SPV, l'analyse a révélé comment les choix linguistiques du locuteur ont une incidence sur la force argumentative du discours: ils entraînent une certaine orientation argumentative et mènent les énoncés vers des conclusions tout en reflétant des appréciations favorables ou défavorables. Pour compléter ces résultats, nous envisageons une étude quantitative de la distribution des mécanismes évaluatifs et des options de gradation de notre corpus. Une étude du système de l'ENGAGEMENT serait aussi très utile pour identifier l'image que la Cour projette d'elle-même et son positionnement par rapport aux points de vue d'autrui. Ces travaux permettraient d'obtenir des données plus précises sur les enjeux discursifs des textes juridiques.

\section{BIBLIOGRAPHIE}

Anscombre Jean-Claude et Ducrot Oswald, L'Argumentation dans la langue, Liège, Mardaga, 1983.

Borja Albi Anabel, « Los géneros jurídicos », in Enrique Alcaraz Varó, Las lenguas profesionales y

académicas, Barcelona, Ariel, 2007, pp. 141-153.

Cabre Maria Teresa, « Textos especializados y unidades de conocimiento : metodología y

tipologización », in Joaquín García Palacios et M. T. Fuentes Morán (ed.), Texto, terminología y

traducción, Salamanca, Almar, 2002, pp. 15-36.

Calsamiglia Helena et Tuson Amparo, Las cosas del decir. Manual de análisis del discurso, Barcelona, Ariel, 1999. 
Ciapuscio Guiomar et Kuguel Inés, « Hacia una tipología del discurso especializado : aspectos teóricos y aplicados », in Joaquín García Palacios et M. Teresa Fuentes Morán (ed.), Texto, terminología y traducción, Salamanca, Almar, 2002, pp. 37-73.

Cunillera Montserrat, « Les formules impersonnelles dans les textes judiciaires français et leur traduction en espagnol », Babel. International Journal of Translation, vol. 58, n 2, 2012, pp. 164-180.

Duarte Carles et Martinez Anna, El lenguaje jurídico, Buenos Aires, A-Z Editora, 1995.

Ducrot Oswald, « Esquisse d'une théorie polyphonique de l'énonciation », in Le dire et le dit, Paris, Minuit, 1984, pp. 171-233.

Gardin Pierre, « Application de la théorie de l'Appraisal à l'analyse d'opinion », MajecSTIC, 2009. Disponible en ligne [http://majecstic2009.univ-avignon.fr/Actes_MajecSTIC_RJCP/MajecSTIC/ articles/1272.pdf ]. Consulté le 15/10/2015.

Gotti Maurizio, «El discurso jurídico en diversas lenguas y culturas : tendencia a la globalización e identidades locales », Signos vol. 41, nº 68, 2008, pp. 381-401.

Hood Susan et Martin James Robert, « Invocación de actitudes : el juego de la gradación de la valoración en el discurso », Signos vol. 38, n 58, 2005, pp. 195-220.

Lehti Lotta, « Le positionnement intersubjectif dans les blogs des Politiciens français. Étude de cas des interrogations ", in Bres Jacques, Nowakowska Aleksandra, Sarale Jean-Marc et Sarrazin Sophie (coord.), Dialogisme : langue, discours (Actes du colloque international 8-10 septembre 2010, Montpellier), 2011, mis en ligne le 10 juillet 2011. Disponible en ligne [http://www.praxiling.fr/ IMG/pdf_Lehti1.pdf]. Consulté le 15/10/2015.

Martin James R. et White Peter R.R., The Language of Evaluation, Appraisal in English, Londres / New York, Palgrave Macmillan, 2005.

Navarro Federico, « Gradación y compromiso en escritura académica estudiantil de humanidades. Análisis contrastivo desde la teoría de la valoración ", Estudios de Lingüística Aplicada vol. 32, n 60, 2014, pp. 9-33.

Raccah Pierre-Yves, « La pista de los puntos de vista : teoría, descripciones y pruebas en semántica », Letras de Hoje ${ }^{\circ}$ 139, 2005a, pp. 23-50.

Raccah Pierre-Yves, « Une description de l'excessivité en sémantique des points de vue », Travaux linguistiques du CERLICO n ${ }^{\circ} 18,2005 \mathrm{~b}, \mathrm{pp} .171-190$.

Raccah Pierre-Yves, « Contraintes linguistiques et compréhension des énoncés : la langue comme outil de manipulation », Entretiens d'orthophonie, Paris, Expansion Formation et Éditions, 2008, pp. 61-90.

Ribas Marta, Alineació de textos jurídics paral lels (català i castellà) : Alguns problemes, Thèse doctorale, Universitat Pompeu Fabra, Barcelone, 2006. Disponible en ligne [http://www.tdx.cat/ handle/10803/7502]. Consulté le 15/10/2015.

Serpa Cecilia, « Significados de probabilidad y fuerza en los textos legislativos », I Vardande. Revista Electrónica de Semiótica y Fenomenología Jurídicas, vol. 1, n 1, 2013, pp. 3-33. Disponible en ligne [http://i-vardande.com/index.php/vard/article/view/107/91]. Consulté le 15/10/2015. 


\section{NOTES}

1. Ce travail s'inscrit dans le projet national de recherche ValTrad (L'évaluation en traduction), $\mathrm{n}^{\circ}$ de référence FFI2013-42751-P, financé par le Ministère espagnol de l'économie et des finances, 2014-2017.

2. Malgré que certains auteurs distinguent entre les concepts de discours et texte (Calsamiglia et Tusón 1999, etc.), dans la présente étude nous utilisons ces termes comme synonymes, en suivant des auteurs comme Cabré (2002), Ciapuscio et Kuguel (2002) ou Ribas (2006), entre autres.

3. La CEDH est une cour créée dans le cadre du Conseil de l'Europe par la Convention européenne des droits de l'homme, pour veiller au respect des droits et libertés garantis par cette dernière. Toute personne s'estimant victime d'une violation par un État des dispositions de la Convention peut porter plainte devant la Cour. [https://e-justice.europa.eu/content_eu_courts-15-fr.do]

4. La jurisprudence élaborée par la CEDH a une grande influence sur le droit européen car les droits fondamentaux garantis par la convention européenne constituent également des principes généraux du droit de l'Union.

5. Source : http://www.echr.coe.int/Pages/home.aspx?p=home\&c=fra

6. Dans la description de quelques exemples, nous ferons référence à des stratégies qui relèvent de l'ENGAGEMENT mais nous n'analysons pas ce système ici pour ne pas dépasser les limites de notre recherche ; il fera l'objet d'étude dans nos travaux ultérieurs. Par ailleurs, certaines études récentes ont traité ce système, par exemple, Gardin (2009), Lehti (2011), Serpa (2013), Navarro (2014), etc.

7. Dans tous les exemples les caractères gras ont été ajoutés.

8. «45. La Cour européenne des droits de l'homme utilise deux critères pour déterminer si une enquête a été effective:

- l'enquête doit permettre de déterminer si le recours à la force était justifié ou non dans les circonstances [...],

- des mesures raisonnables doivent avoir été prises pour assurer l'obtention des preuves relatives à l'incident en question, y compris [...] le cas échéant, une autopsie propre à fournir un compte rendu complet et précis des blessures ainsi qu'une analyse objective des constatations cliniques, notamment de la cause du décès [...]».

9. En espagnol le mode conditionnel exprime un degré d'intensité plus faible que le futur (Serpa 2013 : 17) ; nous considérons que cette affirmation est valable aussi pour le conditionnel en français.

10. Le choix et le degré de l'engagement du locuteur envers les points de vue alternatifs révèlent sa propre vision et ils reflètent également celle qu'il attribue à d'autres locuteurs (Martin et White 2005 : 95-96).

\section{RÉSUMÉS}

Les arrêts relèvent d'un genre juridique, la jurisprudence, qui dans la culture française se veut objectif et impartiel. Cependant, même si le locuteur essaie de cacher sa présence et sa subjectivité, il est obligé de statuer sur un cas et pour ce faire il doit évaluer les allégations du requérant. Nous nous demandons comment l'évaluation est exprimée dans ce type de textes, 
quels sont les mécanismes linguistiques utilisés à cet effet. Le texte choisi pour notre analyse est un arrêt de la Cour européenne des Droits de l'Homme (CEDH) : l'“Affaire Etxebarria Caballero c. Espagne", prononcé le 7 octobre 2014 par la Troisième Section. La requérante saisit la CEDH pour dénoncer la violation de l'article 3 de la Convention européenne des Droits de l'Homme, sur la prohibition de la torture, de la part des autorités espagnoles. Afin de déterminer la nature sémantique des éléments linguistiques mis en place par le locuteur pour construire son évaluation et parvenir à une conclusion favorable ou défavorable pour la requérante, nous appliquerons les systèmes de l'Attitude et de la Gradation de la théorie de l'Appraisal (Martin et White 2005), ainsi que certains instruments conceptuels de la Sémantique des Points de Vue (Raccah 2005).

Judicial decisions belong to a legal genre, case-law, which in French culture seems to be objective and impartial. However, although the speaker tries to hide his presence and subjectivity, he is expected to adopt a position and give a judgement. The aim of this paper is to examine how evaluation is conveyed in these legal documents and which are the linguistic mechanisms used by the speaker to do so. The text chosen for our approach is a judgement issued by the Third Section of the European Court of Human Rights (HUDOC): "Case Etxebarria Caballero v. Espagne" (7th October 2014). The applicant lodges an appeal to HUDOC in order to denounce a violation of Article 3 of the European Convention on Human Rights, which prevents the torture and illtreatment of people in detention or in the custody of the police, by the Spanish authorities. In order to analyze evaluative mechanisms, Appraisal Theory (Martin and White, 2005) is applied to the corpus, as well as some conceptual tools taken from Point of View Semantics (Raccah, 2005).

\section{INDEX}

Mots-clés : sémantisme, jurisprudence européenne, évaluation, gradation, point de vue.

Keywords : semantics, European case-law, appraisal, attitude, point of view.

\section{AUTEUR}

\section{MONTSERRAT CUNILLERA DOMĖNECH}

Universitat Pompeu Fabra 